\title{
Staff Education and Evaluation for Vessel Health and Preservation
}

\author{
Linda J. Kelly
}

\begin{abstract}
Effective teaching and subsequent evaluation of the competence of practitioners is crucial if patient safety is to be assured. Learning is a very complex process, and despite many years of research, it remains difficult to articulate how educational and training methods enable effective learning. We are, however, aware that the actions and attitudes of both the teacher and the learner affect the outcome of the teaching and learning experience. Unfortunately, many individuals teach others without the formal knowledge of how learners learn. Often the teacher lacks the concept to understand, explain and articulate a process during the learning process. This chapter intends to give a general overview of teaching and learning; it develops by exploring and discussing the issue of competency attainment and competency evaluation. Issues of continuous assessment will be considered as will the notion of lifelong learning.
\end{abstract}

\section{Keywords}

Competency · Competency assessment

Education $\cdot$ Evaluation $\cdot$ Adherence to protocols

L. J. Kelly $(\square)$

Edinburgh Napier University,

School of Health and Social Care, Edinburgh, UK

e-mail: Linda.kelly@ vygon.co.uk

\subsection{Approaches to Teaching and Learning}

The training and education of healthcare professionals have changed greatly in recent years. Gone are the days of 'learning on the job' or the 'see one, do one, teach one' method that had been the norm for so many a year. Historically, these were the traditional methods of teaching surgical procedures but raised concerns regarding patient safety. Although this method of teaching has since fallen out of favour, Kotsis and Chung (2013) argue that this method may still be valid if combined with a variety of other adult learning principles. Despite this claim, education and training are generally very structured processes, and academia is typically included. This approach allows the ability to clearly apply theory to practise.

To ensure that education is effective, it is important to have knowledge of some of the major views of learning. In psychology, there are several ideas about how learning occurs. In the twenty-first century, cognitive and social theories were most commonly used, with constructivism (how humans learn) as the most common approach to learning. The approach to teaching and learning clinical skills such as vascular access device insertion and care and maintenance should be underpinned by a constructivism or adult learning philosophical framework such as experiential learning. This is because higher-level 
skills such as those required for vascular access device insertion are generally mastered following repeated experiences of the task. According to Bradley (2006), learners construct their understanding through their interaction with it. A facilitator is required to help practitioners construct their knowledge and skills. Once the theory has been learned, skills are evaluated by the learner using a reflective process which is continuous. Thereafter, learning is constructed from the experience, and practice is improved as evaluation and reflection continue (Hulse 2013).

\subsection{Experiential Learning (Learning by Doing)}

Experiential learning is a model of learning where learning and meaning are derived from repeated patient care experiences. According to McLeod (2012), professional judgement and higher-level skills can be gained from repeated exposure to these experiences. Learning of this type takes place either within a clinical setting or a simulated environment. These learning experiences are designed and supported to ensure that a complete learning cycle is completed. This includes a concrete and tangible experience, observations and reflections, formation of abstract concepts and generalisations, followed by the testing of implications of concepts learned in new situations (Kolb 1983) (Fig. 21.1).

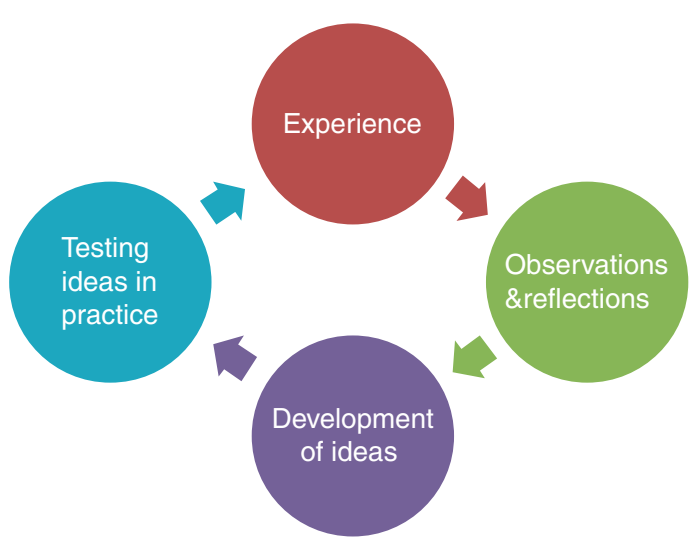

Fig. 21.1 Model of experiential learning process (used with permission L. Kelly)
Theoretical perspective and empirical knowledge are gained from various disciplines including psychology, sociology, ethics, management, education and biological sciences.

The principle of experiential education is a methodology that involves educators or mentors engaging with learners in direct experience. This is followed by reflection which helps the learner to increase knowledge, further develop their skills and clarify values (Caldwell and Grobbel 2013). When applying experiential learning, account must be taken of the learner's previous experience, their knowledge base and their theoretical preparation. An example of this is when an individual has had previous experience of the procedure of peripherally inserted central venous catheter insertion. If service development takes place and this individual's role expands to insert tunnelled central venous catheter, their learning curve will be different to that of someone who had never inserted a central venous catheter. In other words, the learning needs and context of each learner will always vary. This teaching method takes this variation into account, and each educational intervention guides the learner from being dependent towards becoming a participant under supervision, before finally becoming an independent practitioner. This can also be described as the journey from novice to expert (Fig. 21.2) (Benner 1982).

\subsection{Developing Competence: From Novice to Expert}

\subsubsection{Situated Learning}

In contrast to the tradition classroom learning that involves abstract knowledge which is out of context, it is argued that learning should be 'situated' as learners acquire a greater learning experience when knowledge is presented in settings and situations that would normally involve that knowledge (Wacquant 1992). Social interaction and collaboration are important components of situated learning, and learners become involved in a 'community of practice'. As the novice learner moves from the fringes of a 
Fig. 21.2 Novice to expert modified

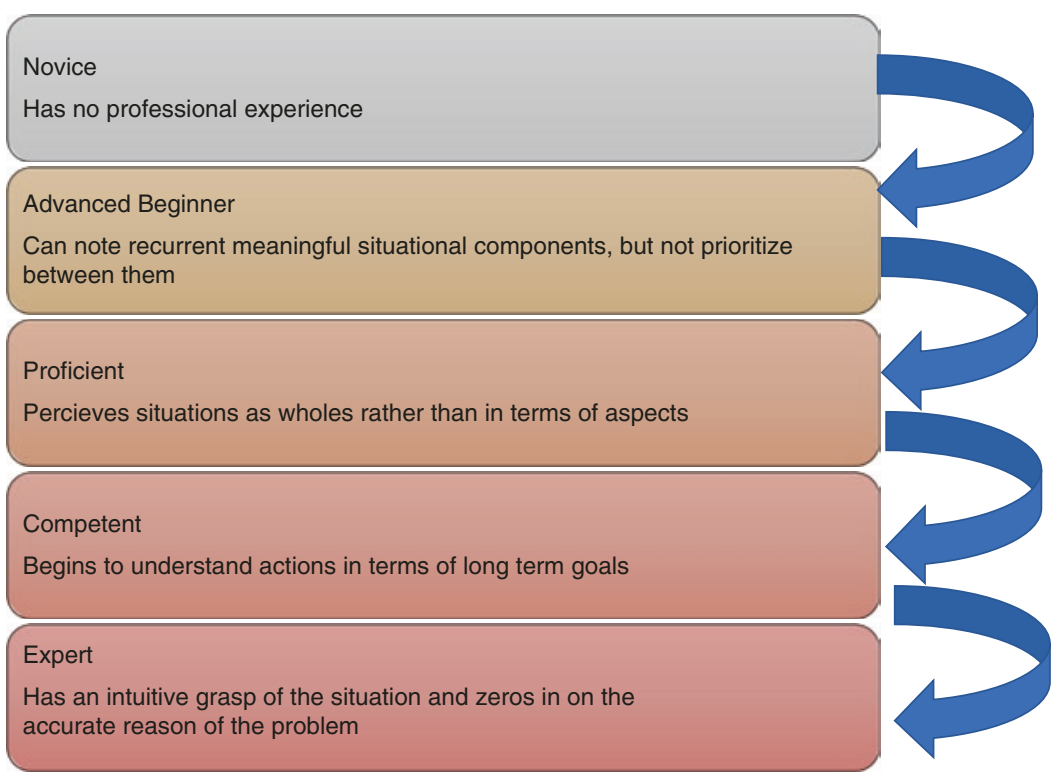

community to its centre, they become more actively engaged in the values and principles and eventually assumes the role of an expert (Collins and Greeno 2010). Within nurse education, simulated care environments are now commonly used to allow learners the opportunity to gain and practise skills in as close to clinical as possible (Onda 2012). Part-task simulation has been recognised as a positive learning tool within vascular access (Kelly et al. 2015). In this learning model, an experienced practitioner or mentor is the most valuable resource available to the learner. To ensure an effective partnership, it should be assured that the mentor - learner relationship is one that is dynamic and active. A positive learning environment is also one that will foster effective learning.

\subsubsection{Situated Cognition and Cognitive Apprenticeship}

The learning of vascular access skills in clinical practice can be characterised as situated learning. Usually, following a period of theory and learning the procedures of ultrasound guidance and device insertions on phantoms and models, nurses will then go into clinical practice to gain confidence and competence in the insertion procedure. Because these skills are being honed in the same or similar environment that the learner will be in once competence is achieve, this could be viewed as situated learning. Cognitive apprenticeship was introduced as an instructional model for situated learning and comprises of six teaching methods to support learning. These methods are:

- Modelling.

- Coaching.

- Scaffolding.

- Articulation.

- Reflection.

- Exploration.

The methods used in this model are very specific and designed to enhance learning in clinical practice. The cognitive processes of the experts play a critical role during complex task performance. They should aim to perform complex tasks in ways that simplifies it for the learner who is observing. This makes it easier for the learner to eventually reproduce the task on their own. Therefore, someone learning the skill of vascular access device insertion should work closely with a mentor and use observation of this competent person to learn skills 
and develop their competence over time. At the time of observation and learning, questioning by the learner and clear explanations from the mentor will help to improve this relationship and provide a positive learning experience.

Suggests that this type of learning results in a highly meaningful situated cognition and enhances the transfer of knowledge to other situations. Situated learning, unlike the more abstract theoretical, preclinical learning, is a more powerful learning experience as it is easier to translate it into concrete situations. In the field of vascular access, there are many models of teaching programmes and courses all aimed at increasing the competency of staff and, ultimately, to ensure positive outcomes (see Chap. 4).

Practitioners in vascular access roles are typically adult learners who are undertaking a programme of study that includes both didactic and practical hands-on practices of the procedure (Jamison et al. 2006). Practitioners in the learning stages should be able to recognise their learning needs, plan ways in which to address these needs and participate in information seeking. Once learning has been completed, it is vital to assess and evaluate this learning to ensure skills attainment, knowledge and understanding.

Fig. 21.3 Basic model of curriculum development (used with permission L. Kelly)

\subsection{Assessment of Learning}

The Quality Assurance Agency (QAA) defines assessment as 'any process that appraises an individual's knowledge, understanding, abilities or skills'. Assessment of clinical practice is essential within the healthcare profession. Competency can be defined as 'possessing the required skills, knowledge, qualifications or capacity', while defines competency as 'the integration of fundamental knowledge, clinical ability, performance, and attitude in the context of a nursing situation'. Competence is usually measured against set criteria and involves the assessment of an individual's performance in relation to a particular standard (Quinn 2000). With regard to vascular access, there is general agreement of what topics should be incorporated into an education and training package for device insertion and subsequent care and maintenance as noted in Chap. 4 (RCN 2016; Moureau et al. 2013).

Once a programme of training has been designed, it is important to ensure that the teaching and assessment are aligned constructively (Biggs 1999) (see Fig. 21.3). According to Biggs, teaching should be a balanced system in which all components support each other. This means that the learning outcomes and choice of assessment must match to allow competency to be

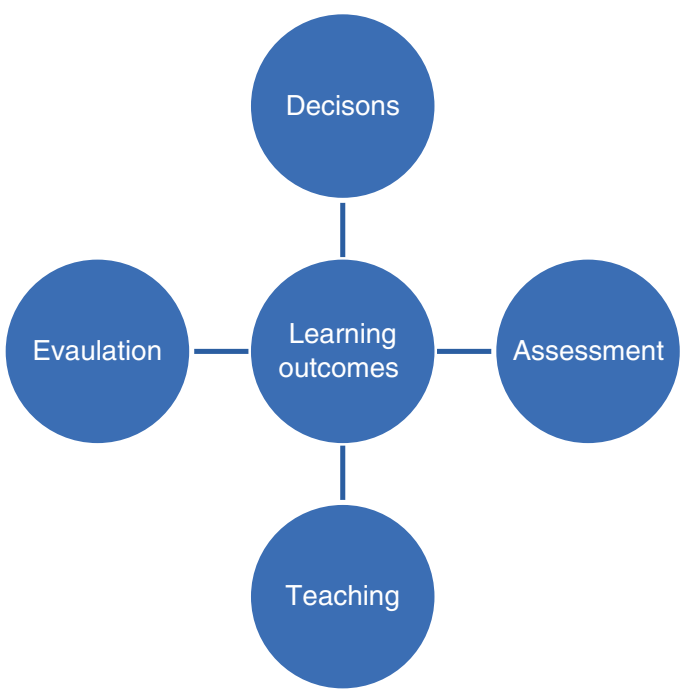


achieved. The teaching, learning and assessment strategies that we participate in must engage in a scholarly manner. To prevent surface teaching, ensure that:

- Learning outcomes are achievable.

- Levels and standards expected at various stages are clearly defined.

- Learning tasks and assessment are aligned.

\subsection{Learning Outcomes/ Alignment to Assessment Methods}

In terms of vascular access insertion education, examples of learning outcomes (LO) and how they can be aligned are provided below:

LO: Demonstrate knowledge of the physiology of blood flow and its importance in device and vein selection.

- Teaching centred on theory, self-directed study and practical sessions using ultrasound.

- Assess knowledge using a variety of methods, e.g. discussion with mentor, written work and diagrammatical explanation, during a patient assessment.

LO: Critically discuss the pathophysiology of thrombosis formation, Virchow's triad and how it is affected by vascular access device selection, insertion technique and location.

- Teaching would be didactic, self-directed study (reading) and practical teaching using ultrasound.

- Assess learning outcome by requiring a written piece of work. This allows student to formulate arguments and develop critical analysis skills.

LO: Demonstrate a skilful, successful ultrasound-guided vein puncture.

- Teaching would involve supervised practice, theory and practice in clinical practice or simulation using part-task phantoms and videos.
- As a practical skill, assess learning outcome using formal visual assessment by the mentor. This could either be done using part-task simulation or direct observation of an insertion procedure.

LO: Identifies and explains the signs and symptoms of arterial puncture and explains immediate interventions.

- Teaching would take place during didactic session, self-directed study (reading) and reflection.

- Assess learning outcome prior to hands-on practice. The assessment could involve discussions with the mentor and could also include some written work to allow for a complete assessment of knowledge and understanding.

\subsection{Clinical Competence: Demonstration, Assessment and Evaluation}

The validation of competency can have a direct impact on patient outcomes. In fact, in today's healthcare, patients expect competent care (Carney and Bistline 2008). Once competency has been acquired, assessment and evaluation of competency is necessary. There are a variety of ways in which clinical competency is demonstrated, assessed and evaluated. However, the most common method of assessing clinical competence is with competency assessment tools (Franklin and Melville 2015). Competency assessment tools have been in use for many years now, particularly within undergraduate programmes (Harris et al. 2010). To ensure that clinical assessments tools accurately measure the competence of learners, it is imperative that the tool be both reliable and valid:

- Validity-The degree to which a measure assesses what it was intended to measure.

- Reliability-The degree to which a score of other measure remains unchanged upon test and retest (when no change is expected). 
The evaluation method must also be objective, observable and measurable. Therefore, it is imperative that those performing the evaluations be trained in using methods such as rater reliability studies that ensure consistency or test/ retest which is a straightforward way of testing the stability or reliability of something. Global rating scales also allow for some degree of consistency in vascular access device insertion. The individual performing the evaluation has a vital role and must ensure the evaluation is performed the same way each time with no variation (Hyrkäs and Shoemaker 2007). It is suggested that standardised documentation for evaluation is useful to help increase compliance with guidelines. Competency should be measurable and evaluated regularly to ensure positive patient outcomes. Competency assessments should, whenever possible, reflect real-life situations such as that achieved in a simulation suite (Butler et al. 2011; Carney and Bistline 2008). It is usually recommended to use more than one assessment tool to produce as accurate an assessment as possible (Franklin and Melville 2015). The way in which competence can be evaluated is by direct observation either in the clinical setting or within simulated environments using part-task training arms.

One of the principal areas of concern regarding the use of clinical competency tools is that we often end up with a 'one-size-fits-all' document. Additionally, the documents produced are generally only able to measure the psychomotor aspects or the 'science' of a procedure but less able to assess the softer 'art' of nursing, such as intuition (Benner 1982). There are many considerations to consider when developing clinical assessment tools. The aim is to have a meaningful tool that is not just a tick box.

To gather this evidence of competence, Boritz and Carnaghan (2003) suggest that rather than a snap shot, the competency assessment be performed on multiple occasions, periodically and across a variety of contexts. The number of multi-faceted approaches to assessment is vast. Wilbeck in 2011 (Wilbeck et al. 2011) identified 11 methods of evaluating competency, namely:
- Benchmarking.

- Checklist evaluation.

- Global rating assessment.

- Simulation.

- Standardised patient.

- Observed Structured Clinical Examination (OSCE).

- Objective Structured Assessment of Technical Skills (OSATS).

- Peer review/rating.

- Portfolio.

- Procedure log.

- $360^{\circ}$ evaluation.

\subsection{Evaluating Staff Competence in Vascular Access Procedures}

Following specific guidelines for vascular access, device insertion can reduce the risk of central line-associated bloodstream infections (Ciocson et al. 2014). Clinicians should adhere to standards and guidelines that have been proven to reduce and eliminate CLABSIs. According to the National Healthcare Safety Network, these standards include:

- Observation: Accountability to ensure inserter is following protocol.

- Experience and comfort of inserter: Inserter must have received special training to insert device.

- Reason for insertion: Is the access device necessary?

- Performance of hand hygiene prior to central line insertion: Basis for aseptic technique.

- Maximal sterile barrier precautions used: Keep sterile fields clear from contamination.

- Skin preparation: Kill bacteria prior to insertion to eliminate infection.

- Selection of insertion site: Healthy vasculature available to deliver therapy.

- Number of lumens: Use least number of lumens necessary to deliver therapy.

Insertion of a vascular access device is viewed as an evidence-based 'high-impact' intervention. 
studied the effectiveness of the care bundle approach to reducing venous catheter-related complications in critical care. In this study, it was proven that adherence to the evidence-based recommendations from the Centre for Disease Control and Prevention (CDC) resulted in a significant decrease in catheter-related bloodstream infections. The low-level evidence base included the following interventions:

- Handwashing.

- Surgical-ANTT.

- Chlorhexidine skin prep.

- Minimise use of femoral approach.

- Remove device when no longer medically necessary.

These interventions led to a dramatic reduction in catheter-related bloodstream infections per 1000 days from 27 to 0 in 3 months. Following this study, a similar programme with an audit has since been rolled out internationally. Therefore, we know that the care bundle approach can be very effective, but compliance to the care bundle is required to ensure this outcome.

\subsection{Evaluation of Competence: Focus on Vascular Access Care Bundles}

Only after being deemed competent is a clinician allowed to perform device insertion without supervision. Often, a combination of methods is used to demonstrate competency. Many institutions continue to base competency on a required number of procedures performed. This approach has been challenged by ACGME, who suggests that it is not simply the number of procedures carried out that is important but that competency should be reviewed using a formal evaluation process. It has been suggested that competency be defined by the percentage of times in which successful independent skills performance is achieved and that an $80 \%$ to $90 \%$ success rate be required prior to independent device insertions.
Intermittent evaluation of the insertion procedure on a regular basis (e.g. annually) is required to ensure competency is maintained. In addition to device insertion, the day-to-day care and maintenance of the device should be carried out in a systematic and evidence-based fashion. Once again, evaluation of the care and maintenance on a regular basis is required to ensure all interventions are carried out.

Overall, competency in vascular access can be demonstrated by four competency assessment steps linking theory to practice:

1. Knowledge of the field of vascular access (devices, relevant anatomy and physiology, care and maintenance, prevention of complications, etc.)

2. Competence in techniques such as 'scrubbing the hub', effective flushing techniques.

3. Performance of skills such as ultrasound guidance, device insertion, etc.

4. Action (Ilic 2009).

In addition to knowledge, skills and attitudes, competence also includes the practitioner's problem-solving abilities, critical thinking skills, decision-making and reasoning skills, as well as excellent communication skills and the ability to work as an effective team member. All of these factors are taken into consideration when determining whether or not a clinician is competent to perform an insertion procedure alone.

Within vascular access, specific individual practices are known to improve care. When these practices are performed together as part of a care 'bundle', they produce a substantially greater improvement of care and reduction in infection rates than when performed indiscriminately. Following a group of practices such as an 'insertion bundle' or a 'care and maintenance' bundle ensures patient safety as well as successful implementation of the VHP framework.

A key principle of care bundles involves the level of adherence to the bundle; clinical variation should be avoided unless there is clinical indication for it, as the bundles have been established 
through evidence-based studies and are proven to reduce patient risks. Variation should never be the result of passive omission. Elements of the bundles should be implemented in every patient $100 \%$ of the time. Compliance of adherence to the bundle is of utmost importance. It must be assured that every step of the bundle is capable of being audited - it was completed, or it was not completed. Please note, the audit will only assess that the intervention was completed and not how well or poorly this was done. This information is often recorded in a simple tick box. If all aspects of the bundle are completed, it can be said that the care bundle has been completed. Occasionally, due to good reason such as patient allergy, aspects of a care bundle may be omitted. If there is not a good reason for omission of one or more steps in a care bundle, this can be seen as a failure of care.

\subsection{How Can We Improve Compliance to Care Bundles?}

- Validate evidence supporting bundle practices while making the information available to clinicians adopting the practices.

- Increase compliance with the introduction of care bundle checklists or end-of-bed charts.

- Make care bundle compliance part of a quality marker with the inclusion of a financial implication.

\subsection{Lifelong Learning}

In addition, as professionals we are responsible for continuously updating our practice and must now consider the notion of lifelong learning (Numminen et al. 2013). Adaptive practitioners should be committed to lifelong learning. Once a skill is acquired and competency met, there should be mechanisms put in place to ensure that competence is maintained, and the individual remains competent and therefore safe enough to continue to perform the procedure.

\subsection{Summary}

The right competency assessment ensures that practitioners are adequately prepared and safe to perform procedures or care for patients. Vascular access has changed vastly over the past two decades, and we are now aware of the many factors that ensure vessel health. The right approach to teaching and learning is the first step in this process. Following education, it is vital that the learning is assessed and that the right knowledge and skills have been gained. Competency is then achieved and validated using a recognised and effective method or tool. Finally, learning and competence should not be viewed as a one-off event; lifelong learning should become the norm.

\section{Case Study}

Jane French was delighted to be offered a staff nurse role within a busy vascular access service. One of the key roles would be to insert peripherally inserted central catheters (PICCs). Following her general orientation to the role, she began to focus on the procedure of device insertion.

Jane was given a competency document to guide her through the process. She was teamed up with a mentor, Oliver, who would act as her teacher and supervisor. She was instructed to approach any team member at any time with questions and queries if Oliver was unavailable.

Jane was supplied with an in-depth competency framework to guide her through her learning. This consisted of:

- Reading up on anatomy and physiology of arm veins as related to vascular access.

- Physiology of blood flow.

- Information on device selection.

- Patient assessment.

- Infection prevention and control.

- Ultrasound guidance. 
- Device insertion techniques.

- ECG tip location device information and use.

- Complications (prevention, recognition and management).

Each of these sections contained reflective sections, further reading and questions to ensure learning had taken place.

In addition, Jane spent time in a simulation suite and put her learning into practice. With the use of part-task simulation, she gained practice and skills in:

- Ultrasound-guided venous access using phantoms.

- PICC insertion using a simulated model.

- Use of ECG tip location device.

- Dressing and flushing.

Once confident, she spent time working alongside Oliver, first, in an observational stance and then performing the procedure under supervision with Oliver scrubbed up with her. Jane reflected on each procedure performed and wrote this up in her competency document.

The number of supervised insertions was decided between Jane and Oliver, and this continued until Jane deemed herself competent and felt comfortable with the procedure and when Oliver agreed with her decision. The final competency sheet was signed by both parties to indicate competency had been attained.

Jane and Oliver continued to meet regularly and discuss her progress and to discuss any issues or concerns. Periodic competency assessment was integrated as part of the facility policies.

After 2 months, Jane was practising independently but continued to reflect and build her knowledge and skills in vascular access device insertion.

\section{Summary of Key Points}

1. Providing the right approach to teaching and learning is the first step in preparing competent practitioners.

2. Following education, it is vital that learning is assessed on a regular basis to ensure that the right knowledge and skills have been gained.

3. Competence assessment using a recognised and effective method or tool ensures that practitioners are adequately prepared to perform procedures or care for patients.

4. As professionals, we are responsible for continuously updating our practice. We therefore need to commit to lifelong learning. Learning and competence should not be viewed as a one-off event.

\section{References}

Benner P. From novice to expert. Am J Nurs. 1982;82:402-7. https://doi.org/10.1097/01.NUMA.0000313089.04519. $\mathrm{f} 4$.

BiggsJ.Assessingforlearningquality:II.Practice.In:Teaching for quality learning at University; 1999. p. 165-203. https://doi.org/10.1097/00005176-200304000-00028.

Boritz JE, Carnaghan CA. Competency-based education and assessment for the accounting profession: a critical review*. Can Account Perspect. 2003;2(1):7-42. https://doi.org/10.1506/5K7C-YT1H-0G32-90K0.

Bradley P. The history of simulation in medical education and possible future directions. Med Educ. 2006;40:254 62. https://doi.org/10.1111/j.1365-2929.2006.02394.x.

Butler MP, Cassidy I, Quillinan B, Fahy A, Bradshaw C, Tuohy D, O'Connor M, Mc Namara MC, Egan G, Tierney C. Competency assessment methods-tool and processes: a survey of nurse preceptors in Ireland. Nurse Educ Pract. 2011;11(5):298-303. https://doi. org/10.1016/j.nepr.2011.01.006.

Caldwell L, Grobbel C. The importance of reflective practice in nursing. Int J Caring Sci. 2013;6(3):319-26. International Journal of Caring Sciences SeptemberDecember 2013; Vol 6 Issue 3.

Carney DM, Bistline B. Validating nursing competencies using a fair format. J Nurs Staff Dev. 
2008;24(3):124-8. https://doi.org/10.1097/01.NND. 0000300881.48802.b7.

Ciocson MAFR, Hernandez GM, Atallah M, Amer SY. Central vascular access device: an adapted evidence-based clinical practice guideline. J Assoc Vasc Access. 2014;19(4):221-37. https://doi. org/10.1016/j.java.2014.09.002.

Collins A, Greeno JG. Situated view of learning. In: International encyclopedia of education; 2010. p. 335-9. https://doi.org/10.1016/B978-0-08-044894-7.00504-2.

Denton A. Standards for infusion therapy. Royal College of Nursing; 2016. p. $41 \mathrm{t} / \mathrm{m}$ 42. Publication code: 005704.

Franklin N, Melville P. Competency assessment tools: an exploration of the pedagogical issues facing competency assessment for nurses in the clinical environment. Collegian. 2015;22(1):25-31. https://doi. org/10.1016/j.colegn.2013.10.005.

Harris P, Snell L, Talbot M, Harden RM. Competencybased medical education: implications for undergraduate programs. Med Teach. 2010;32(8):646-50. https:// doi.org/10.3109/0142159X.2010.500703.

Hulse AL. Clinical competency assessment in intravenous therapy and vascular access: part 2 . Br J Nurs. 2013;22(17):1008-13. https://doi.org/10.12968/ bjon.2013.22.17.1008.

Hyrkäs K, Shoemaker M. Changes in the preceptor role: re-visiting preceptors' perceptions of benefits, rewards, support and commitment to the role. J Adv Nurs. 2007;60(5):513-24.

Ilic D. Assessing competency in evidence based practice: strengths and limitations of current tools in practice. BMC Med Educ. 2009;9(1):53. https://doi. org/10.1186/1472-6920-9-53.

Jamison RJ, Hovancsek MT, Clochesy JM. A pilot study assessing simulation using two simulation methods for teaching intravenous cannulation. Clin Simul Nurs. 2006;2(1):9-12. https://doi.org/10.1016/j. ecns.2009.05.007.

Kelly LJ, Green A, Hainey K. Implementing a new teaching and learning strategy for CVAD care. Br J Nurs. 2015;24(8):S4-8, S9, S12. https://doi.org/10.12968/ bjon.2015.24.Sup8.S4.
Kolb D. David A. Kolb on experiential learning. 1983. www.infed.org/biblio/b-explrn.htm. https://doi. org/10.1177/0255761407088489.

Kotsis SV, Chung KC. Application of see one, do one, teach one concept in surgical training. Plast Reconstr Surg. 2013;131(5):1194-201. https://doi.org/10.1097/ PRS.0b013e318287a0b3.

McLeod S. Bruner-learning theory in education I simply psychology. Simply Psychology. 2012. http://www. simplypsychology.org/bruner.html.

Moureau N, Lamperti M, Kelly LJ, Dawson R, Elbarbary M, Van Boxtel AJH, Pittiruti M. Evidence-based consensus on the insertion of central venous access devices: definition of minimal requirements for training. Br J Anaesth. 2013;110(3):347-56. https://doi. org/10.1093/bja/aes499.

Numminen O, Meretoja R, Isoaho $\mathrm{H}$, Leino-Kilpi H. Professional competence of practising nurses. J Clin Nurs. 2013;22(9-10):1411-23. https://doi. org/10.1111/j.1365-2702.2012.04334.x.

Onda EL. Situated cognition: its relationship to simulation in nursing education. Clin Simul Nurs. 2012;8(7):e273-80. https://doi.org/10.1016/j.ecns. 2010.11.004.

Quinn F. Curriculum theory and practice. In: Principles and practice of nurse education; 2000. p. 131-51. http://medcontent.metapress.com/index/ A65RM03P4874243N.pdf\%5Cn; http://books.google. com/books?hl=en\&lr=\&id=qILGb7xcXFIC\&oi=fnd $\& p g=P P 1 \& d q=$ Curriculum + theory + and + practice $\&$ ots $=\mathrm{MuS} 4 \mathrm{sZlKmu \& sig=2-a0BmsV34nUfqpi4pFMsFxD}$ $\mathrm{SgU}$.

Wacquant L. Cognition in practice: mind, mathematics, and culture in everyday life. Actes de la recherche en sciences sociales. 1992; https://doi. org/10.2307/2073537.

Wilbeck J, Murphy M, Heath J, Thomson-Smith C. Evaluation methods for the assessment of acute care nurse practitioner inserted central lines: evidence-based strategies for practice. J Assoc Vasc Access. 2011;16(4):226-33. https://doi.org/10.2309/ java.16-4-5.

Open Access This chapter is licensed under the terms of the Creative Commons Attribution 4.0 International License (http://creativecommons.org/licenses/by/4.0/), which permits use, sharing, adaptation, distribution and reproduction in any medium or format, as long as you give appropriate credit to the original author(s) and the source, provide a link to the Creative Commons license and indicate if changes were made.

The images or other third party material in this chapter are included in the chapter's Creative Commons license, unless indicated otherwise in a credit line to the material. If material is not included in the chapter's Creative Commons license and your intended use is not permitted by statutory regulation or exceeds the permitted use, you will need to obtain permission directly from the copyright holder.

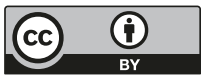

